\title{
The Presentation of Self and Identity Construction in Virtual World: A Case Study of Avakin Life
}

\author{
Lisa Vangohol Jande \& Noor Aireen Ibrahim* \\ Language Academy, Faculty of Social Sciences and Humanities, Universiti Teknologi Malaysia, 81310 UTM \\ Johor Bahru, Johor, Malaysia
}

Submitted: 29/09/2020. Revised edition: 29/11/2020. Accepted: 29/11/2020. Published online: 30/12/2020

\begin{abstract}
Identity in the virtual world has become a point of interest for researchers. The virtual world has become a place where people go to interact and in turn create an identity for themselves. This study sets out to investigate identity construction in a $3 \mathrm{D}$ Virtual world game using Avakin Life as a case study. Going with Goffman's theory of self-presentation, this study aims to understand why identities are created in the virtual world and how this identity is created. The process to achieving this is carried out through a review of previous literature on virtual world identity construction and an ethnographic research carried out in Avakin Life using observation and semi-structured interviews as a method for data collection. The data is analysed thematically, and the findings show that participants use the virtual world to create an extension of themselves while they achieve this creation by duplicating a piece of themselves through the use of their Avatars. This study therefore helps to add to the existing knowledge on identity and identity construction in the virtual world.
\end{abstract}

Keywords: Virtual world, identity, identity construction, avatar, self-representation, avakin life

\subsection{INTRODUCTION}

The virtual world can be a fertile land for diverse identity. People have greater freedom to build and display other facets of their identities, especially in the more anonymous virtual settings (Turkle, 1995). With virtual game players having liberty over the construction of identity in the virtual world, they therefore, have the ability to control the way people view their identity. The ability to implement and maintain numerous online identity-exposition techniques allows the player generally to be able to provide more personal information about themselves in a computer-mediated environment were anonymity is guaranteed (Madell \& Muncer, 2007).

Various research has been conducted on identity and the virtual world (e.g. Ecenbarger, 2014, Bullingham \& Vasconcelos, 2013, Turkle, 1994). However, most of these research focus on the impact of identity construction on players and not how or why this identity is created. Also, several research (e.g. Bessière, Seay \& Kiesler, 2007; Diehl \& Prins, 2008) can be found on identity in the

\footnotetext{
*Correspondence to: Noor Aireen Ibrahim (email: naireen@utm.my)
} 
virtual world but often centred on Second Life or the World of Warcraft. Few, if any, research exists with regard to Avakin Life and yet, most are focused on language used in the virtual world (Zein, 2017, Amri, Tanduklangi \& Alberth, 2016). Hence, based on Goffman's (1959) theory of selfrepresentation as a framework, this study seeks to answer the question; why do people construct identity in the virtual world? How is this identity constructed? In addition, this study also seeks to add to the existing knowledge on virtual world and identity construction, making reference particularly to Avakin Life as a case study from an ethnographic perspective.

\subsection{Avakin Life (AL)}

Avakin Life (AL) is an online 3D virtual world game produced by Lockwood publishing since 2013. At the time the research was conducted, AL was recorded as having more than 50, 000, 000 (fifty million) downloads and about 500,000 daily active users (https://avakin.com/about/). AL provides a player with the opportunity to be whoever they wish to be. The players can choose their avatar referred to as Avakin in AL and customize it as they wish. There are several methods of customization such as skin tone, hairstyle, and hair colour. For the female avatars, the aspects of customization are the facial features such as shape of the face, eyes, nose, eyebrows, mouth and ears. In addition, other customizations include facial makeup, jewelleries, tattoos, clothes and shoes, animations ranging from standing pose to dance moves, actions that have to do with jumping, flying, and seating. For the male avatar, the customization is similar to the female avatar as mentioned above, with addition of facial hair such as beards of different types and color.

Another aspect of AL is the ability of players to own and furnish their own accommodation (e.g. apartment or house) and purchase cars. In addition, players can also travel to different community structures within AL ranging from game communities where players can meet to play games, social spots where players can go to socialize, like clubhouses, open apartments, hotels, and dance studios. There are also some available locations where players can go find jobs and earn money.

An avatar can work and earn money to be converted to Avacoin, which is a type of currency used only within AL to buy anything an avatar wants in the virtual world. There are shops where an avatar can purchase clothing, ranging from expensive designer wear to unbranded wear. In addition, players may also purchase facial and body features. Pets are also available for purchase ranging from animals like cats, dogs, fantasy animals, wild animals, and farm animals.

\subsection{LITERATURE REVIEW}

This study attempts to capture previous research on virtual world and identities in virtual worlds. The purpose of this section is to discuss what virtual world means and the identity construction in these worlds. It also explores other researcher's opinions on identity in the virtual world. 


\subsection{Definition of Virtual World}

Virtual world or virtual reality has been defined differently by different researchers. A virtual world is a synchronic, continuous human network, depicted as avatars, made easier by networked computers (Bell, 2008). According to Dionisio, Burns, and Gilbert (2013), a virtual world is a Computergenerated 3-dimensional entity or system simulations with apparently real, direct, or physical user interaction. Dionisio et al. (2013) went further to elaborate that a virtual world is a persistent online computer-generated space where multiple users can communicate or function in real-time in distant physical locations for work purposes or play. Going by the afore-mentioned definitions, the virtual world is a multi-element integration which according to Muhanna (2015) includes, "computers, worlds and environments, interactivity, immersion, and users, who are usually referred to as participants in a virtual reality experience" (346).

Within the virtual world, users use their avatars to communicate with each other and the environment. Avatar according to Song \& Jung, (2015) refers to an electronic image representing the user and also being manipulated by the user. Most scholars interpret avatars as an extension of self into the virtual world (Evans 2016, Animesh, Pinsonneault, Yang, \& Oh, 2011, Ratan \& Hasler, 2010).

\subsection{Goffman's Theory of Self-presentation}

According to Erving Goffman (1959) identity is dynamic. Goffman considers identity and selfpresentation as a performance which changes depending on the situation the performer finds himself. Identity can, therefore, be structured based on how an individual presents himself. Goffman (1959) in his work "The Presentation of Self in Everyday Life" using the theatre to demonstrate contrasting actions of individuals classifies this performance into two parts namely the front stage and the backstage. Front stage being where the performer adopts or create for themselves an identity to suit the performance and backstage is when the performer can be himself. Goffman also refers to the "splitting" character of the self, a situation in which individuals decide what to bring to the front stage and what to reserve in the backstage and in some situations the backstage persona is brough into the front stage.

Goffman further uses the term impression management to refer to individuals' performance on stage. Individuals portray themselves in a certain position and influences the impression they give, thus also affecting the view created in the minds of the audience. According to Goffman (1959), this is done in order to meet the expectations of the audience and the social norms in order to receive positive review. The participating individual therefore attempts to show their "idealised" self and to portray themselves in the best possible light. Often, those positive or appealing facets of their personality are exaggerated while ignoring or fully concealing the negative ones.

This can be compared to the identity representations found in a virtual world such as Avakin Life. The players use this virtual world as their stage and create identities of themselves that conform to the expectations of intended audience or to fulfill a certain aim or task the players wish to accomplish. It is with this understanding that this study seeks to examine why individuals construct a particular identity and in what way the construction of the identity is managed. 


\subsection{Identity and Identity Construction in the Virtual World}

Identity tends to be the result of a combination of personal and social attributes that creates for each personality a specific configuration (Parmentier \& Rolland, 2009). This goes to say that every individual is identified either by features that expose their participation in one or more social groups (social identity) or by more personal attributes that are distinctive (personal identity) (Hogg, Terry \& White, 1995). There is a strong link between social identity and personal identity in that they become a vital part of an individual through interaction. According to Parmentier \& Rolland (2009) it, therefore, seems that identity can be generated or rebuilt by means of a dual psychological process "(1) a process of emotional closure, a result of others' reactions to mobilized role identities, leading to available role identities being fixed; (2) a process of imaginative opening up, creation of possible selves, fed by the individual's experiences and imagination, leading to the creation of new role identities when interacting with others" (p.5)

Scholars have long realised the importance of identity in the digital environment (Donath, 1999). In the virtual world, identity is noticeable by an avatar. A player can select an avatar and customize it the way he or she deems fit, and most often, this goes in line with the player's identity. A player may decide to select blond hair for their avatar because she has always had a preference for blonde locks, or she may select black hair because she has always admired how black hair looks on other people. In this instance, the hair colours of the avatar are related to the identity of the individual and therefore the avatar is seen as part of an extension of the person's self (Ratan \& Hasler, 2010).

Virtual world games like Avakin Life can help to promote a strong connection between the player and his/her avatar because the game closely depict real life and players adopt avatars to be a representation of themselves in this virtual world. For instance, during the course of this ethnography research in $\mathrm{AL}$, the researcher had a conversation with an avatar who used his real name for his avatar. In other words, with the realness of the virtual world and the interaction that avatars experience within the virtual environment, it is easy for them to depict their real self thereby having the avatars becoming more or less the embodiment of themselves, leading in an incredibly realistic virtual experience (Animesh et al., 2011). Therefore, virtual worlds like Avakin Life try to create many aspects of reality precisely as it is in real life, thereby giving rise to the term "Virtual reality." On the other hand, other online games, in comparison, can show more differing views from real life since such games are already free of the simulation of real-life (Song \& Jung, 2015).

\subsection{The Virtual World as a Place to Explore Identity}

Like social networking, virtual worlds present avenues for learning and social interaction, especially for those who find it difficult to be expressive in a face to face communication (Warburton, 2009; Falloon, 2010). The anonymity provided by the virtual world makes it easy for users to express themselves confidently, which also encourages them to be expressive and experience new things or identities (Heikoop, 2013).

According to Antonacci et al. (2008), the virtual world is an environment that allows and, in many ways, encourages social interaction and personalities to evolve. Antonacci et al. went further to 
mention that some interactive innovations in the virtual world to be perpetual, for example, the growing social engagement among individuals and communities; the focus on individual and group identity as well as the enhanced sense of presence through an avatar. This goes to say that the virtual world provides an avenue for users to explore. Virtual worlds give users numerous possibilities with the knowledge that personality creation and presentation is dynamic and contextual (Heikoop, 2013). Based on Messinger et al. (2008), Vasalou et al. (2008), Kafai et al. (2010) and Martey \& Consalvo (2011), the three reasons why players create avatars in the virtual world the way they do is because of "(i) to embody aspects of their 'real' selves; (ii) to present an idealized self; and (iii) to explicitly challenge off-line norms and behaviour" (as cited in Heikoop, 2013, p.59). This means that some of the players who build avatars in virtual worlds choose to represent them with certain elements of themselves in real life. These players also want to give specific attributes to their avatars that they cannot exhibit in real life (Kafai, Fields, \& Cook, 2010). For instance, dressing their avatars in particular ways, or portraying their avatars with a hairstyle they cannot typically put on in real life. This can be referred to as an extension of themselves because even those who decide to choose or customize avatars to look younger than their real-life person is only seen as idealising their real self (Heikoop, 2013).

The virtual world creates the capacity for players to express themselves in ways they cannot easily do in face to face conversations, which are often how "identity placement" is created (Heikoop, 2013). Bargh, McKenna \& Fitzsimons (2002) in their research on expression of the 'True Self' on the Internet, the findings showed that the virtual world is mainly used by individuals to convey the best parts of themselves: "the phenomenally significant and objectively true facets of self that are not communicated often or readily to others" (p. 34). They discovered that people found it easier to show aspects of themselves that could not be expressed in real life such as being a great dancer or exhibiting certain aggressive aspects of themselves that are viewed as a normality in the virtual world but not in real life. The ability to create identity allows players the flexibility and the potential to become "virtually" the ideal person they cannot wholly experience offline (Heikoop, 2013).

\subsection{METHODOLOGY}

This study was conducted based on a qualitative methodology carried out through an ethnographic examination over a three months' period in the virtual world of Avakin Life. Due to the short period of time spent on the study, it may be more accurate to refer to this study as a mini ethnographic examination. This involved being submerged in AL as an observer participant (Kawulich, 2012). This gave the researcher the opportunity to observe and interact with AL participants in order to gather data to answer the researcher questions outlined in this study.

The researcher kept a detailed log of her participation in AL. Initially, online observation was conducted daily for about two hours every day from $10 \mathrm{am}$ to $12 \mathrm{pm}$. However, there appeared to be very few participants during these hours as people were not always available. As a result, the observation period was altered and thus the online logging pattern changed to between $10 \mathrm{pm}-12 \mathrm{am}$ daily and this yielded better results. 
The participants were recruited at random by asking the players if they would be willing to be interviewed in this study. The aim was to get both male and female participants involved in the study. All participants were informed of the objective of the interview and the study. From the 10 participants the researcher approached three agreed to be interviewed. Out of the three participants interviewed, two were female. All discussions were conducted through AL chat medium, which may be challenging as some responses were not immediate, some were very delayed. The interviews were semi-structured and thus the sequence in which the questions were asked varied between the three participants. Often, the interviews will begin with basic questions about how they start playing AL, who introduced them and how often they are online.

During the ethnographic immersion, all interviews were documented and later transcribed. Thematic analysis (TA) adopted from Braun \& Clarke (2012) was used to analyse the data. According to Braun \& Clarke (2012), TA is a method for systematically identifying, organizing, and offering insight into patterns of meaning (themes) across a data set. Thematic analysis was used in this study because the purpose of this study was to find out the reason for identity construction in the virtual world by how the players described their avatars and to try to identify what similarities their avatars had with their real-life personality.

\subsection{Analysis}

First, the interview conducted was transcribed verbatim, and thematic analysis adopted from Braun \& Clarke (2012) was carried out. Braun \& Clarke identified six phases of conducting thematic analysis: i) Familiarizing Yourself with the Data; ii) Generating Initial Codes; iii) Searching for Theme; iv) Reviewing Potential Themes; v) Defining and Naming Themes; and vi) Producing the Report. Following these steps in analysing the data from the interview, the transcribed data was inspected several times, and notes were taken, and the process of coding was also carried out. Codes were colourcoded with Microsoft Word, followed by the categorisation of the highlighted text. This process was repeated until, the following themes emerged, which are i) Identity Extension, ii) Identity duplication and iii) Safety in anonymity.

\subsection{Ethics of the Research}

To observe ethical guidelines as a researcher, the suggestion by Kozinets (2002) was adhered to. Kozinets highlighted four points of code of ethics for ethnographers to follow while conducting research in the virtual world:

- The virtual community should be fully informed about the presence, affiliations, and intentions of the researcher.

- The confidentiality and anonymity of the participants should be ensured.

- The researcher should seek and incorporate feedback from members of the virtual community.

- The researcher should obtain the permission of the participants to use specific postings. 
These were the steps followed in gathering the necessary data for the analysis.

\subsection{FINDINGS AND DISCUSSIONS}

As a result of the analysis of the data collected from AL, three prevailing themes emerged, which assisted in answering the research questions. This section illustrates the findings in the form of a set of verbatim accounts based on the analysed data.

\subsection{Identity Extension}

Identity extension is a process in which a player sees his avatar as an extension of himself and passes some of his personal characteristics to the avatar. For example, his avatar may bear similar physical resemblance to the player's real self while adding additional features or attributes the player may wish to acquire or have in real life. This goes in line with Goffman's (1959) notion of impression management where participating individual creates their "idealized" self to portray themselves in the best possible light, in this case they want to create the best version of themselves.

This first theme, therefore, address the question of why virtual world players construct their particular identity. The virtual world offers a platform to create a version of oneself that in the physical world is hard to achieve (Bullingham \& Vasconcelos, 2013), and this in itself becomes an extension of the player's identity. The physical attributes of the avatar are partly the same as that of the player in the physical world, but with an addition of other attributes or characteristics the player does not have or cannot exhibit in real life. For instance, one of the participants had this to say.

Researcher: Tell me about your Avatar?

Avatar Jodi: I got inspired by my avatar from like billie eilish style I know it sounds stupid, but I really liked how her personality was, but I didn't want to be her in as the type of way when you look exactly like her, but I wanted to have her personality and her style but instead of her face and everything else I wanted me to be in her characteristics."

Billie Eilish is an American teenage singer whose sense of style can been considered unusual with equally uncommon make up, and this is the kind of character a player wants to emulate in the virtual world but may not be able to do so in real life. According to Kafai et al. (2010), players choose to give certain attributes to their avatars that they cannot exhibit in real life.

The virtual world offers a platform to create a version of oneself that in the physical world is hard to achieve, and for this reason, a player creates an extension of himself in the virtual world through the use of an avatar. This is in agreement with Heikoop's (2013) perception of identity creation. Heikoop attributes the reasons behind identity creation in the virtual world as a result of players wish to present an idealised self. Idealised self here refers to a desire to be more, and for the players on $\mathrm{AL}$, the inability to accomplish a certain reality in real life seems to motivate players to achieve it in the 
virtual world, extending that part of their identity to their avatar as seen from the interview excerpt below

Researcher: Why did you dress your avatar that way?

Avatar Cherish: I love fashion and I have my way when it comes to dressing up characters on games."

Researcher: Are you a fashion designer in real life?

Avatar Cherish: aspiring to be someday."

For this particular player, she may not be able to be a fashion designer in real life yet, but AL provides her a platform where she can explore that part of her, in turn, creating an extension of her identity through her selected avatar.

\subsection{Identity Duplication and Safety in Anonymity}

Identity duplication refers to a situation in which a player creates an avatar to mirror his offline character in every sense. Identity duplication comes into play because of the safety of being anonymous in a virtual world as no one really knows the true person at the backstage. In identity duplication, a player portrays his avatar in the same way he would appear in real life. This is what Goffman (1959) considers as "splitting" character of the self. Where an individual decides on what to bring to the front stage and what to reserve in the backstage which in this case the players are seen to duplicate themselves through avatars. This helps to answer the question of how identities are constructed.

Avakin life players construct identity by transferring their real-life physical attributes to their avatars. For instance, a player has an avatar that is physically close to his appearance in the physical world. This individual brings his entire self to the virtual world by transferring his real-life physical attributes to his avatar. An example can be seen from the following interview excerpt;

Researcher: What resemblance does your avatar have with you in real life?

Avatar Tusher: hair colour, built, skin tone and name."

Researcher: So Tushar is your real name?

Avatar Tusher: Yes."

Researcher: Why use your real name?

Avatar Tusher: Nobody knows it's my real name anyway until I tell them."

Apparently, this player transfers his real-life features by giving his avatar similar features like his physical self and using his real name. For him, the virtual world, like the physical world, is a place to live in. For these players, duplicating themselves on $\mathrm{AL}$ does not pose a problem because the anonymity in the virtual world provides a sense of protection as shown in the following response:

Researcher: Does your avatar look like you in real life?

Avatar Cherish: She has my skin tone, the hair colour and eyes, and lips. 
Researcher: Why structure your avatar that way when you could be anybody you wish to be on Avakin?

Avatar Cherish: You should show people the real colour of you and they might not know that's what you really look like so doesn't matter."

For players on AL, the anonymity provided by the virtual world makes it comfortable for them to duplicate themselves online without any fear or worry, and this is again in line with Heikoop's (2013) notion that the anonymity provided by the virtual world makes it easy for users to express themselves confidently. Because of this anonymity, players actually feel free to bring a part of themselves and use that in creating their avatar in the virtual world.

\subsection{CONCLUSION AND FUTURE RECOMMENDATION}

This study provides some insights into identity creation in virtual world. Players use virtual worlds and avatars as tools for the creation of identity. Participants use the virtual world to create an extension of themselves while they achieve this creation by duplicating a piece of themselves through their avatars. This study also revealed that virtual worlds are digital spaces where human experiences and personality development are performed. However, it may not be possible to generalise and to conclude that everyone in the virtual world uses the platform as a place for identity construction. What is evident from the data analysed in this study, the virtual world can indeed serve as a place for individuals to be whoever they wish to be without the restrictions found in real life. This study therefore helps to add to the existing knowledge on identity construction in virtual world.

There is a need, however, for more research in this area as there are limitations to the current study that future researchers should take into consideration. Although, there is very little a researcher can do to verify the data collected as the researcher depended solely on the participants response to the interview questions, different sources of data collections may enhance similar future studies. Future research may include both online and offline data collection, for example, examining participant's social media accounts or conducting face to face interviews. Another constraint is the number of participants involved in the current study. More participants may yield more interesting or even contrasting results.

\section{REFERENCES}

Amri, C., Tanduklangi, A., \& Alberth, A. 2016. Integrating Virtual World into Project Based Learning through Teaching and Learning Writing. Journal of Language Education and Educational Technology (JLEET). 1(2). 
Animesh, A., Pinsonneault, A., Yang, S. B., \& Oh, W. 2011. An Odyssey into Virtual Worlds: Exploring the Impacts of Technological and Spatial Environments on Intention to Purchase Virtual Products. MIS Quarterly. 35(3): 789-810.

Antonacci, D., DiBartolo, S., Edwards, N., Fritch, K., McMullen, B. \& Murch-Shafer, R. 2008. The Power of Virtual Worlds in Education: A Second Life Primer and Resource for Exploring the Potential of Virtual Worlds to Impact Teaching and Learning. Angel Learning.

Bell, M. 2008. Toward a Definition of 'Virtual Worlds. Journal of Virtual Worlds Research. 1(1): 1-5.

Bargh, J. A., McKenna, K. \& Fitzsimons, G. M. 2002. Can You See the Real Me? Activation and Expression of the 'True Self' on the Internet. Journal of Social Issues. 58(1): 33-48.

Bessière, K., Seay, A. F., \& Kiesler, S. 2007. The Ideal Elf: Identity Exploration in World of Warcraft. Cyberpsychology \& Behavior. 10(4): 530-535.

Braun, V., \& Clarke, V. 2012. Thematic Analysis. APA Handbook of Research Methods in Psychology. 2(P): 57-71.

Bullingham, L., \& Vasconcelos, A. C. 2013. 'The Presentation of Self in the Online World': Goffman and the Study of Online Identities. Journal of Information Science. 39(1): 101-112.

Diehl, W. C., \& Prins, E. 2008. Unintended Outcomes in Second Life: Intercultural Literacy and Cultural Identity in a Virtual World. Language and Intercultural Communication. 8(2): 101-118.

Dionisio, J. D., Burns, W. G., \& Gilbert, R. 2013. 3D Virtual Worlds and the Metaverse: Current Status and Future Possibilities. ACM Computing Surveys(CSUR). 45(3): 34.

Donath, J. S. 1999. Identity and Deception in the Virtual Community. In P. Kollock \& M. Smith (Eds.). Communities in Cyberspace (pp.29-59). London: Routledge.

Ecenbarger, C. 2014. The Impact of Video Games on Identity Construction. Pennsylvania Communication Annual. 70(3): 34-50.

Evans, S. 2016. Where is the Virtual Self? Virtual Worlds and the Self as a Cyborg. Annual Review of Cyber Therapy and Telemedicine. 14(Summer): 51-57.

Goffman, E. 1959. The Presentation of Self in Everyday Life. New York: Doubleday.

Heikoop, W. 2013. Blended Identities: Identity Work, Equity and Marginalization in Blended Learning. E-Learning and Digital Media. 10(1): 53-67.

Hogg, M. A., Terry, D. J., \& White, K. M. 1995. A Tale of Two Theories: A Critical Comparison of Identity Theory with Social Identity Theory. Social Psychology Quarterly. 255-269.

Kafai, Y. B., Fields, D. A. \& Cook, M. S. 2010. Your Second Selves: Player-Designed Avatars. Games and Culture. 5(1): 23-42.

Kozinets, R. V. 2015. Netnography. The International Encyclopedia of Digital Communication and Society, 1-8.

Madell, D. E., \& Muncer, S. J. 2007. Control Over Social Interactions: An Important Reason for Young People's Use of the Internet and Mobile Phones for Communication? Cyberpsychology \& Behaviour. 10(1): 137-140.

Muhanna, A. M. 2015. Virtual Reality and the CAVE: Taxonomy, Interaction Challenges and Research Directions. Journal of King Saud University - Computer and Information Sciences, $27(3): 344-361$. 
Parmentier, G., \& Rolland, S. 2009. Consumers in Virtual Worlds: Identity Building and Consuming Experience in Second Life. Recherche et Applications en Marketing (English Edition). 24(3): 4355.

Ratan, R., \& Hasler, B. S. 2010. Exploring Self-Presence in Collaborative Virtual Teams. PsychNology Journal. 8(1): 11-31

Song, H., \& Jung, J. 2015. Retracted: Antecedents and Consequences of Gender Swapping in Online Games. Journal of Computer-Mediated Communication. 20(4): 434-449.

Turkle, S. 1994. Constructions and Reconstructions of Self in Virtual Reality: Playing in the MUDs. Mind, Culture, and Activity. 1(3): 158-167.

Turkle, S. 1995. Life on the Screen: Identity in the Age of the Internet. New York: Simon \& Schuster.

Zein, F. 2017. Abbreviation Words Used in 'Avakin Life 3d Virtual World'Online Game Chatting Room. 


\section{APPENDIX 1}

Sample of semi- structured interview questions

1. How did you hear about Avakin Life?

2. How often do you come online?

3. Has it been fun playing so far?

4. Tell me about your Avatar?

5. Is there any particular reason why she/he appears that way?

6. Question here depends on answer in 5(for example are you a fashion designer in real life)

7. does your avatar look like you in real life?

8. why structure your avatar that way when you could be anybody you wish to be on Avakin?

9. What is your opinion of virtual games?

10. Why partake in a virtual game? 